\title{
La face cachée de l'ancestralité. Masques et affinité chez les Matis d'Amazonie brésilienne
}

\section{Philippe Erikson}

\section{OpenEdition}

1 Journals

Édition électronique

URL : https://journals.openedition.org/jsa/510

DOI : $10.4000 /$ jsa. 510

ISSN : 1957-7842

Éditeur

Société des américanistes

\section{Édition imprimée}

Date de publication : 5 janvier 2004

Pagination : 119-142

ISSN : 0037-9174

\section{Référence électronique}

Philippe Erikson, «La face cachée de l'ancestralité. Masques et affinité chez les Matis d'Amazonie brésilienne », Journal de la Société des américanistes [En ligne], 90-1 | 2004, mis en ligne le 05 janvier 2009, consulté le 04 septembre 2022. URL : http://journals.openedition.org/jsa/510 ; DOI : https:// doi.org/10.4000/jsa.510 


\title{
LA FACE CACHÉE DE L'ANCESTRALITÉ MASQUES ET AFFINITÉ CHEZ LES MATIS D'AMAZONIE BRÉSILIENNE
}

\author{
Philippe ERIKSON *
}

\begin{abstract}
Cet article montre en quoi les masques matis sont révélateurs des conceptions ouestamazoniennes de la temporalité et de la succession des générations. Après une discussion sur l'aspect cérémoniel des mascarades et sur les caractéristiques ontologiques imputées aux esprits mariwin, ce texte soutient que ces derniers, bien qu'associés aux morts du groupe et à des valeurs endogènes, représentent des affins virtuels plutôt que des ancêtres. Est également évoquée la possibilité qu'aient autrefois existé des liens rituels privilégiés, aujourd'hui révolus, avecl'ethnie voisine des Katukina-Kanamari. [Mots CLÉs : Amazonie, ancêtres, masques, eschatologie, rituel, alliance, contact interethnique.]
\end{abstract}

Faces from the past : masks, ancestors and affines of the Matis (Amazonas, Brazil). This paper shows how Matis masks reveal Western Amazonian conceptions of temporality and generational continuity. After a discussion of the ceremonial aspects of the masquerade and of the ontological characteristics attributed to mariwin spirits, it suggests that the latter represent virtual affines rather than ancestors, despite their intimate links with the group's deceased forebears and endogenous values. Considerations about the possibility of former preferential ritual relations with the neighboring Katukina-Kanamari are also presented. [KeY woRDS : Amazonia, ancestors, masks, eschatology, ritual, alliance, interethnic relations.]

A cara escondida da ancestralidade. Máscaras e afinidade entre os Matis (Amazonas, Brasil). Este artigo mostra como as máscaras matis ilustram as concepções oesteamazônicas da temporalidade e da continuidade geracional. Após uma exposição da dimensão cerimonial do baile de máscaras e das características ontológicas atribuídas aos espíritos mariwin, defende-se a idéia de que estes últimos representam afins virtuais antes do que antepassados, apesar da íntima ligação que os une com os mortos do grupo e com valores endógenos. Considerações sobre a possível existência no passado de relações rituais privilegiadas dos Matis com os vizinhos Katukina-Kanamari são também apresentadas. [PALAVRA CHAVE : Amazonas, antepassados, máscaras, escatologia, ritual, aliança, contato interétnico.]

* Université Paris X-Nanterre, Laboratoire d'ethnologie et de sociologie comparative [erikson@mae.u-paris10.fr].

Journal de la Société des Américanistes, 2004, 90-1, pp. 119-142. CSociété des Américanistes. 
Dans toute la littérature ethnographique consacrée aux Matis, les personnages masqués que les locuteurs de cette langue pano d'Amazonie brésilienne appellent mariwin ont été dépeints comme la figuration d'esprits qualifiés d'" ancestraux ». À la lumière de nouveaux matériaux de terrain ' , j'aimerais préciser ce qu'il convient d'entendre par " ancestral » dans ce contexte amazonien. Je me propose de montrer que les mariwin accomplissent l'exploit paradoxal d'incarner les valeurs de l'ancestralité tout en se voyant dénier le statut de parents, l'idée même de filiation étant ostensiblement rejetée dans les discours et les pratiques qui les concernent.

Après une discussion sur l'aspect cérémoniel des mascarades et quelques considérations sur les caractéristiques ontologiques imputées aux mariwin, je défendrai l'idée qu'ils représentent l'émanation d'une époque révolue, qu'ils sont distinctement associés aux morts du groupe et aux générations précédentes, ainsi qu'à l'affirmation de soi, mais sans pour autant cesser d'apparaître comme des affins virtuels. Nous verrons au passage que l'affinité en question pourrait être repérée dans l'espace et le temps, puisqu'elle suggère l'existence de liens révolus mais néanmoins privilégiés avec l'ethnie voisine des Katukina-Kanamari. J'espère ainsi apporter un éclairage nouveau sur les conceptions de la temporalité et de la succession des générations qui caractérisent les systèmes de pensée ouest-amazoniens.

\section{LES MARIWIN : ASPECTS RITUELS}

Les mariwin interviennent lors de mascarades au cours desquelles des hommes les personnifient en s'enduisant le corps de boue (parfois teintée de charbon), en se parant de fougères et se recouvrant le visage d'un élégant masque d'argile (Erikson 2002). Ils portent des faisceaux de longues baguettes faites de palmes de daratsintuk (Astrocaryım muru muru), destinées à fouetter les enfants (Figure 1) ; ils se déplacent essentiellement à croupetons, émettant des vocalises et adoptant des mimiques et des postures hautement conventionnelles. Bien que l'aspect ludique (pour ne pas dire touristique) des mascarades semble aujourd'hui gagner en importance, incarner les mariwin en revêtant un masque constitue néanmoins un acte sérieux. On est en effet censé se transformer en esprit et, pour en parler, on utilise d'ailleurs la même expression linguistique (tsusin impak-, " devenir esprit ») que pour se référer au destin post-mortem d'une personne récemment décédée. Se travestir en mariwin, du moins traditionnellement, n'est pas sans danger potentiel pour les acteurs et conduit à prendre un certain nombre de précautions rituelles, notamment pour ce qui concerne le regard (Erikson 2001).

Les mariwin se rendent chez les humains à intervalles irréguliers mais sans périodicité fixe, si ce n'est que leur visite doit normalement coïncider avec la 


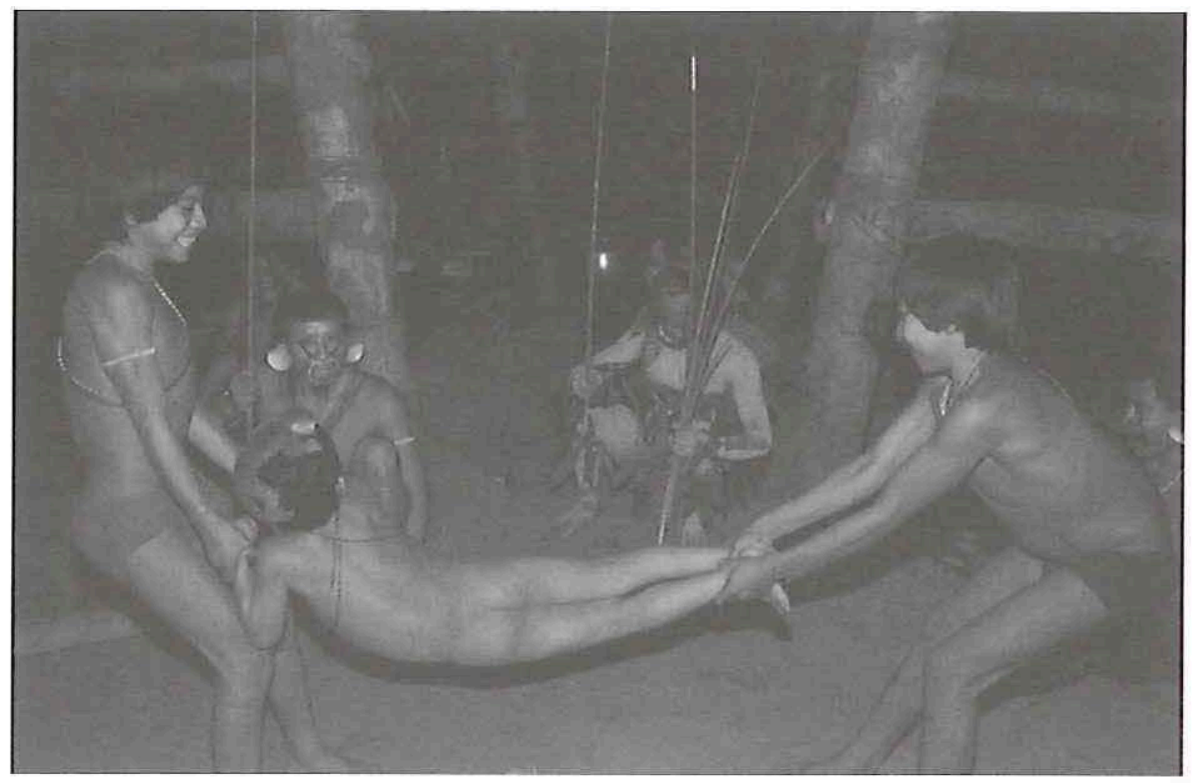

FIG. 1 - Mariwin ocre en action (cliché Erikson)

saison du maïs. Ils viennent par petits groupes de deux ou trois lorsqu'il s'agit simplement de châtier des enfants désobéissants (ce qui est une de leurs fonctions) et en plus grand nombre pour des rituels importants, en particulier l'imposition des tatouages. Lors de ces occasions solennelles, auxquelles il ne m'a jamais été donné d'assister, le groupe des mariwin se lève pour chanter, au lieu de se contenter de grogner, comme il le fait en temps ordinaire. Mariwin et tatouages sont systématiquement associés dans les discours des Matis. Ces derniers ne manquent jamais de faire remarquer que l'ornementation surabondante des masques, pourvus de nombreux labrets de kapok et de plumes d'ara, constitue un modèle qu'ils s'efforcent d'imiter par le biais de leurs propres pratiques ornementales.

Quoiqu'inopinée du point de vue des enfants, l'arrivée des mariwin est bien entendu prévisible pour et même programmée par les adultes. Les femmes sollicitent leur venue par des cris suraigus très codifiés, tandis que les hommes les appellent en jouant du masën, trompe traversière d'argile destinée à cet effet. Les Matis affirment que cet instrument de musique, le seul dont ils disposent, ne doit être joué que lorsque la viande abonde, sans doute parce qu'il s'agit là d'une des conditions sine qua non de leur hospitalité. À l'époque où les Matis vivaient 
encore dispersés, le masën servait également pour convier les habitants des autres villages. On peut en déduire que les mariwin, bien qu'ils ne soient pas nourris, ont un statut d'invités.

La principale fonction des mariwin consiste donc à fouetter les enfants, les adolescents et les femmes enceintes dont la progéniture bénéficie indirectement des coups, pour ainsi dire par métonymie. Loin de relever de la punition, les coups sont plutôt un moyen de transférer de l'énergie (sho) à ceux qui les reçoivent. Même s'ils servent accessoirement à sanctionner la méconduite des jeunes, ils sont avant tout perçus comme un outil pour stimuler la croissance et remédier à la paresse, de sorte que les adolescents, à la différence des plus jeunes qu'il faut parfois contraindre, s'y exposent bien volontiers. D'ailleurs, selon les Matis, les badines des mariwin agissent moins en vertu de leur caractère cinglant - incontestable au vu des marques qu'elles laissent sur la peau - que par les innombrables (et minuscules) épines dont elles sont pourvues. Cette multitude de piquants permet aux « esprits » d'injecter; au sens le plus littéral du terme, une partie de leur énergie (sho) aux récipiendaires. Avant de les utiliser, les mariwin enduisent parfois les baguettes d'un peu de leur sueur, matérialisation concrète de l'énergie qu'ils cherchent à transmettre ${ }^{2}$. Retenons en outre qu'on s'impose, pour garantir leur efficacité, d'obtenir les badines « à la sueur de son front », en les déterrant à mains nues, plutôt qu'en les coupant avec une machette.

Au cours des mascarades, tout en restant accroupis et en se déplaçant dans une démarche chaloupée qui les caractérise, les mariwin brandissent d'un geste saccadé la base de leurs badines, manifestant ainsi leur intention de " piquer» (sek) les enfants (Figure 2). Aucun geste ne signifie leur volonté de les fouetter; preuve supplémentaire qu'on est bien dans une logique d' " acupuncture ». Les enfants peuvent observer cette gestuelle stéréotypée, que l'on serait tenté de qualifier de danse. Ils peuvent également profiter du spectacle des pantomimes que les mariwin effectuent pour répondre aux questions plus ou moins saugrenues que les humains leur adressent pour l'amusement général. En revanche, au cours de la cérémonie, on leur rappelle fréquemment qu'ils doivent éviter de regarder les esprits, en particulier au moment crucial où le fouet s'apprête à tomber sur le bas de leur dos ou le haut de leurs cuisses (selon l'effet désiré). Cela se justifie pratiquement par le risque de recevoir un coup de fouet dans l'œil. On ne peut cependant s'empêcher de rapprocher cet interdit oculaire de la contrainte visuelle, symétrique et inverse, à laquelle sont soumis les mariwin qui doivent constamment fixer les enfants du regard. Une fois leur visite terminée, c'est à reculons qu'ils sortent de la maison afin de ne jamais quitter les enfants des yeux. Nous reviendrons sur ce point important, car il fait penser à certaines croyances pano relatives aux composantes immatérielles des défunts.

Les enfants les plus jeunes, au lieu d'être fouettés, ont juste le pied caressé par un paquet de plantes médicinales (bwate) pour les encourager à marcher. Cette pratique suscite des commentaires insistants sur l'endurance des mariwin, dont 


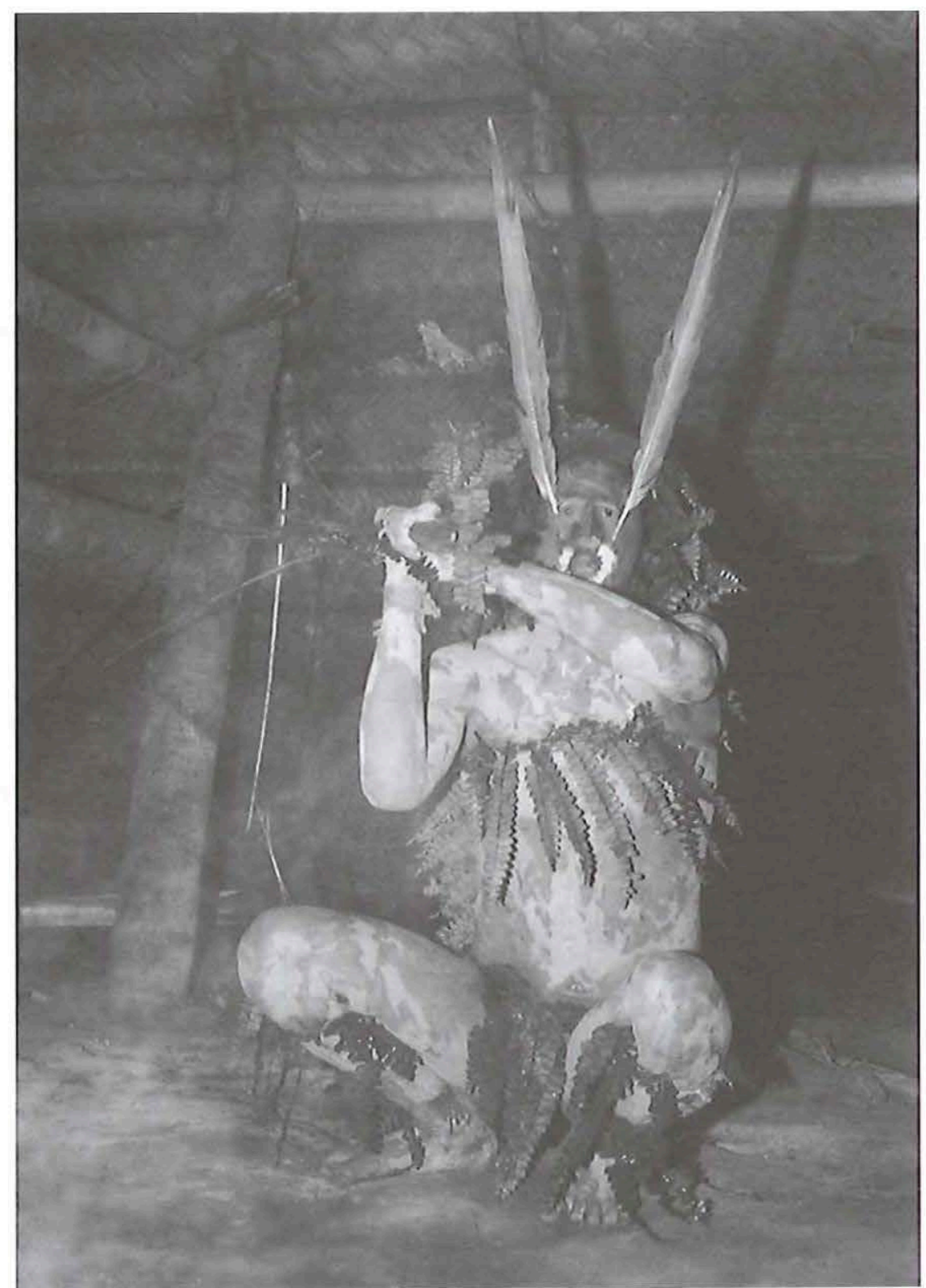

Fıg. 2-Gestuelle stéréotypée d'un Mariwin : posture de menace (cliché Erikson) 
on se plaît à signaler qu'ils viennent de très loin, des anciens jardins des villages aujourd'hui abandonnés ou des falaises localisées en aval, sur les berges des fleuves. On a montré ailleurs que l'aval représentait une direction systématiquement associée à la mort, à l'altérité et au passé (Erikson 1989).

Les femmes, et surtout les enfants, sont supposés ignorer que ces personnages masqués sont des humains déguisés plutôt que de "véritables » mariwin. Les femmes adultes, en particulier celles de la catégorie des macho (femmes ménopausées), feignent seulement d'être dupes, puisqu'elles font partie intégrante du rituel. Ce sont elles qui préparent le fond de poterie sur lequel chaque homme rajoutera des traits faciaux pour le transformer en masque. Elles fabriquent le support que les hommes se contentent de décorer. Elles interviennent aussi dans le déroulement de la mascarade en proférant des cris rituellement performatifs dont l'analyse indique sans ambiguïté qu'elles savent pertinemment de quoi il retourne (Erikson 2000a).

Aujourd'hui, en raison du grand nombre de performances ad hoc réalisées pour satisfaire la curiosité des visiteurs occasionnels (cinéastes, touristes, représentants de la FUNAI ou de diverses ONG), le spectacle des mariwin s'est nettement engagé sur la voie de la manifestation folklorique, avec toutes les conséquences que l'on peut imaginer. Les enfants ne se laissent plus abuser; les acteurs sont de plus en plus jeunes, diverses innovations ont touché la forme des masques, la nature des grognements et l'agencement même du "show ». Il suffit pour s'en convaincre de comparer les descriptions de ce que j'ai observé au milieu des années 1980 avec, par exemple, ce qui a été récemment mis en scène pour (et diffusé par) Discovery Channel, TV Globo, Rai Uno, Fuji Television ou Canal Plus ${ }^{3}$. Les mariwin n'ont de secret que pour l'ethnologie, car tant les enfants matis que les téléspectateurs occidentaux en savent plus que jamais sur leur compte. Jusqu'à la fin des années 1980, les Matis cachaient jalousement leurs masques. Aujourd'hui, ils en produisent en grand nombre pour la vente et certains interlocuteurs vont jusqu'à nier l'existence de « vrais » mariwin, indépendamment des représentations qu'en font les humains (en revanche, tout le monde croit fermement en l'existence des esprits maru dont on reparlera plus loin). Néanmoins, si le rituel a été quelque peu galvaudé dernièrement, les croyances cosmologiques qui sous-tendent les mariwin demeurent vivaces. Voyons à présent ce qu'il en est de ces mariwin conceptuels qui semblent mieux résister à la modernisation que leurs avatars rituels.

\section{LES MARIWIN : DIMENSION ONTOLOGIQUE}

Dans l'imaginaire matis, il existe trois catégories de mariwin, chacune associée à une couleur, à un oiseau et à un écotype. On distingue les mariwin kuru (de couleur ocre-jaune), les mariwin put (rouges) et les mariwin wisu (noirs). Les 
premiers s'appellent aussi « shiashkegit », tandis que les deux derniers (surtout les noirs) peuvent être qualifiés de " winu winu tsusi », expression que l'on peut traduire par " esprits des instruments de bois de palmier Bactris ». Une érudite matis, Chiampi Teshkam, m'a expliqué que les mariwin noir et rouge étaient en fait chuka (" homonymes ») l'un de l'autre, qu'ils relevaient donc d'une seule et même catégorie - le lien d'homonymie ayant ici une force toute particulière (Erikson 1993). Il m’a été précisé que les mariwin rouges étaient plus jeunes que les noirs, ce que semble confirmer le symbolisme des couleurs, puisque le noir renvoie à l'ancienneté chez les Matis, tandis que le rouge est, comme partout en Amazonie, lié à tout ce qui est neuf. N'ayant appris l'existence des mariwin kuru (ocres) qu'en 1996, je les ai confondus avec les mariwin put (rouges) dans bon nombre de mes publications antérieures, dans lesquelles j'affirmais que les rouges habitaient dans les palmeraies des anciens villages, tandis que les noirs demeuraient dans des falaises riveraines. Il faudrait reprendre l'enquête sur ce point, afin de vérifier si ce ne seraient pas les ocres plutôt que les rouges qui résident dans les jardins abandonnés. Quoi qu'il en soit, les mariwin sont chacun associés à un type d'ara différent, dont on les dit « kurasek », terme qui signifie « jaloux » ou, plus exactement, « qui entretient une relation privilégiée avec » (ce qui n'est évidemment pas incompatible avec la jalousie). Ainsi, les mariwin ocres sont kurasek des aras jaunes, les rouges des aras rouges et les noirs des bleus.

Au-delà de leurs différences, les trois sortes de mariwin ont en commun un certain nombre de caractéristiques relatives à leurs préférences alimentaires, à leur mode de perception et à leur morphologie. Les mariwin se nourrissent tous d'aras (jeunes, m'a-t-on spécifié) qu'ils chassent à la sarbacane (ils n'ont pas d'arc) et dégustent accompagné de mamõ ( "résine ») ${ }^{4}$. Cependant, en fonction d'un de ces chambardements perceptifs (ou changement de focale) auquel l'ethnologie amazoniste s'est beaucoup intéressée ces dernières années, les mariwin considèrent la résine (qui est noire) comme du manioc doux (qui est blanc) et les aras (dont la viande est obscure et dure) comme du kwëbu (Penelope jacquacu, oiseau dont la viande est blanche et tendre). Qu'on y voit un effet perspectiviste ou l'avatar indigène d'une opposition structurale, toujours est-il que ce qui est noir et dur pour les humains semble blanc et tendre aux esprits. On pourrait pousser le raisonnement plus loin en posant que ce qui fait la vieillesse pour les simples mortels connote au contraire la jeunesse pour ces êtres, lesquels, en matière de séniorité, voire d'ancestralité, ont plusieurs longueurs d'avance. Ce qui semble très vieux à un jeune peut paraître très jeune à un vieux et cela se traduit dans le code chromatique utilisé pour parler de ces hyper-aînés que sont les mariwin.

Dans une optique complémentaire de la première, on peut interpréter ces paradoxes perceptifs en y voyant le reflet d'un principe d'inversion. La vision des mariwin - êtres de l'inframonde car ils viennent du sous-sol - est présentée comme l'envers, sinon le négatif, de celle qui nous est familière. Par exemple, pour 
ce qui concerne leur morphologie, une des caractéristiques principales des mariwin est précisément d'avoir le devant derrière et le dedans dehors (et viceversa). Outre qu'ils avancent souvent à reculons lors des rituels, on dit en effet que les mariwin, à l'inverse des humains, ont le winte (terme pour "cœur» ou " pouls ") à l'arrière du crâne, de sorte qu'ils pourraient mourir si on les frappait là, alors que leur face est au contraire extrêmement dure (et, de fait, le masque ne couvre que le devant du visage, étant juste attaché derrière) ${ }^{5}$. De plus, on dit que leurs innombrables labrets traversent directement leurs os, et non pas leur peau et leur chair, comme si leur charpente osseuse était extériorisée à la manière d'une carapace. Ici encore, le contraste est total avec ce qui se passe chez les humains, dont les ornements sont piqués dans les parties molles du corps plutôt qu'à même le squelette ${ }^{6}$.

Notons que les mariwin ont des dents apparentes sur le masque (en fait, elles correspondent à leurs labrets), mais qu'ils sont aussi censés en disposer de vraies tout au fond de leur tête ("ukëmuruk»), dans ce qu'on pourrait appeler la "sous-face ». Bien que parfois représentées sur certains masques atypiques par des dents de pécari, ces " vraies dents » sont comparées à celles des caïmans et unanimement qualifiées d'isamarap ( «affreuses »). Au lieu de les avoir saillantes et tournées vers l'extérieur - comme il sied à ces instruments de prédation -, celles des mariwin sont donc « intériorisées » ${ }^{7}$.

Parmi les traits communs à tous les mariwin, quelle que soit leur couleur, l'un des plus remarquables est qu'ils sont tous très étroitement reliés au maïs et aux palmiers Bactris, l'association étant conjoncturelle dans le premier cas et franchement symbiotique dans le second. J'ai montré ailleurs la nature quasi consubstantielle du lien qui unissait les palmiers Bactris - la toute dernière plante à produire des fruits dans un abattis - et les esprits mariwin (Erikson 2001). La relation qu'ils entretiennent avec le maïs est de nature différente, puisque cette plante est attachée à la jeunesse, rôle qui lui convient merveilleusement car elle est la toute première à pousser dans un abattis neuf. De plus, on ne la cultive qu'en prévision de rituels, dans une ambiance qui confine au cérémoniel. Les Matis disent parfois que les mariwin sont tsari kurasek, " jaloux-proches du maïs » (plante que l'on récolte au milieu de la saison des pluies, comme les Bactris). D'après Tumi Preto, cette expression (souvent entendue) signifie que les mariwin se fâcheraient si les humains ne finissaient pas tout le maïs de leurs propres jardins et redoubleraient alors la force de leurs coups. Toujours selon Tumi Preto, les mariwin habitent dans leurs propres abattis, sur les berges des fleuves, mais viennent régulièrement dans les jardins des humains, où on les trouve souvent au pied des arbres brûlés (là où se plantent les palmiers Bactris). Leur présence garantirait une meilleure croissance du maïs. L'ethnologue ne peut s'empêcher de voir une illustration supplémentaire du parallèle omniprésent dans le symbolisme rituel matis entre le destin biologique du maïs et celui des candidats au tatouage : leur croissance est également tributaire d'une cérémonie dont le succès exige la 
venue en nombre des mariwin. Tumi Preto m'expliqua que les mariwin viennent nombreux à condition que les femmes regardent vers le haut, et non pas vers les souches, lorsqu'elles récoltent le maïs. Il peut arriver cependant qu'elles (ou des enfants) découvrent un masque dans un jardin abandonné ou récemment brûlé. Il est alors justifié comme étant les restes d'un mariwin qui aurait péri dans un incendie. Les mariwin sont mortels à leur manière et peuvent avoir des enfants. Une fillette d'une dizaine d'années m'a signalé qu'en saison sèche, les mariwin ne venaient pas parce qu'il n'y avait pas de maïs et qu'ils étaient morts. L'avis n'émane peut-être pas d'une experte, mais n'en est pas moins frappant par sa grande vraisemblance par rapport à tout ce que l'on sait par ailleurs sur ces esprits.

Ayant désormais une meilleure idée de ce que sont les mariwin, il nous reste à voir ce qu'ils ne sont pas ou, plus exactement, ceux dont ils semblent représenter l'antithèse parfaite : les esprits maru.

\section{L'OPPOSITION MARUIMARIWIN}

Éminemment dualiste, la typologie matis des êtres repose sur des séries oscillant entre deux pôles, respectivement qualifiés d'ayakobo et de tsasibo, dont le premier connote l'altérité et le second l'intériorité. Bien que son rendement sociologique soit aujourd'hui extrêmement modeste, cette polarité évoque clairement un système de moitiés avec l'une du « dedans » et l'autre du « dehors » dont on trouve de nombreux exemples dans toute l'aire pano (Townsley 1987). D'innombrables indices, provenant de divers domaines, laissent entendre qu'un système dualiste de ce type opérait encore chez les Matis dans un passé relativement récent (Erikson 1996). Dans cette perspective, la catégorie des mariwin, qui relève des tsasibo (moitié du dedans), s'oppose à celle des maru, qui appartient aux ayakobo (moitié du dehors).

À première vue - mais on verra qu'il faut nuancer ce propos - le contraste entre mariwin et maru est total ; ces deux types d'esprits se différencient comme le social et l'asocial, le visible et l'invisible, l'hirsute et le chauve, le bénéfique et le malfaisant, pour reprendre des thèmes traité ailleurs (ibid.). Si les mariwin incarnent la figure prototypique des valeurs matis (endurance, générosité, absence de paresse, forte teneur en sho) et exhibent une ornementation corporelle " hyperbolique ", les maru se caractérisent au contraire par leur malignité (ils manifestent une propension à égarer les gens en forêt) et une absence totale d'ornementation et de pilosité, ce qui est censé les rendre invisibles. Les premiers constituent une sorte de modèle que les générations contemporaines s'efforceraient bien vainement d'égaler, tandis que les seconds - souvent plus ridicules que véritablement détestables et comparés volontiers aux curupira lors de conversations avec des Néo-brésiliens - apparaissent plutôt comme un repoussoir. 
En première approximation, l'opposition maru/mariwin évoque donc incontestablement celle entre ayakobo et tsasibo. Cependant, comme c'est très généralement le cas en Amérique indigène, un dualisme peut en cacher un autre ; c'est bien ce qui se passe ici puisque les différents mariwin se répartissent entre ayakobo et $t$ sasibo. Plus précisément, les mariwin noirs et rouges sont qualifiés de $t$ sasibo, tandis que les mariwin jaunes (mariwin kuru) seraient pour leur part ayakobo. On est visiblement en présence d'un dualisme réduplicatif, en forme de poupées russes, tel qu'il a maintes fois été décrit pour l'aire andine, mais dont on trouve bien des équivalents dans les basses terres (Molinié 1988). Cette légère " pointe » d'ayakobo qui semble transparaître chez les mariwin va de pair avec l'allure grotesque des quelques rares masques de mariwin pourvus de dents «laides » (artefacts atypiques; voir Erikson 1996, p. 276). Ces faits suggèrent une paradoxale conjonction entre les catégories, pourtant opposées, de mariwin et de maru, illustration supplémentaire, si besoin était, du caractère fort peu manichéen du dualisme amérindien. Mariwin et maru s'excluent et se supposent. Ils se définissent l'un par rapport à l'autre mais jamais l'un sans l'autre.

Les maru ont une dimension asociale, voire anti-sociale, mais - reprenant une piste suggérée par Bidou (1999, p. 80) dans un texte consacré aux homologues de ces esprits en Amazonie du Nord-Ouest - on pourrait aussi les envisager comme " pré-sociaux », jaloux des humains parce qu'ils seraient semblables à des enfants qui n'ont pas encore reçu de corps. De fait, chez les Matis, les nouveau-nés leur sont assez souvent comparés : en tant que glabres et encore dépourvus d'ornements, on utilise volontiers à leur encontre l'expression maru maru pa, « similaires aux maru ». Quoi qu'il en soit, on constate que de nombreux éléments formellement proches des productions de l'activité humaine, mais sans en avoir l'utilité, sont attribués aux maru, en particulier un grand nombre de végétaux sauvages qui évoquent des artefacts ou des plantes cultivées. Les portions de forêt abattues par le vent sont nommées marun maë (« jardins des maru»), les bananiers sauvages sont marun tsinkwin (« bananes des maru »), les grands champignons blancs qui poussent sur les arbres en décomposition sont dits marum paut (« les pendants d'oreille des maru ») ${ }^{8}$, etc. À l'exception, ô combien prévisible, du maïs, pratiquement toutes les plantes cultivées ont une contrepartie sauvage imputée aux maru. Or tous ces produits se caractérisent par leur imperfection et/ou leur caractère improductif, comme pour mieux souligner que ce qui concerne les maru frise l'humanité, mais sans jamais l'atteindre pleinement.

La danse au cours de laquelle les hommes matis imitent les maru est une variation sur ce thème. Munis de bâtons grossiers censés représenter des couteaux géants, les danseurs prennent en effet un malin plaisir à tout détruire dans la maison commune (notamment les poteries) en essayant d'aiguiser leurs « lames » de bois. Ils chantent dans la langue ésotérique imputée aux maru: " tambos menas, shëpi menas, kwen mas menas, awane menas ", ce qui signifie que leurs haches ne sont pas affûtées et qu'ils ont donc bien du mal à couper des arbres. De 
fait, si les mariwin entretiennent un lien étroit avec les abattis des humains, les " abattis des maru », en revanche, ne sont guère que des petites clairières abattues par le vent (remarquons qu'un lien étroit unit les maru au vent).

Bien que les maru soient théoriquement invisibles, Iba Shono et son frère Kwini ont néanmoins pu me les décrire avec précision, après m'avoir assuré que d'autres Matis (la défunte Dani-Macho, entre autres), en avaient réellement rencontré. Ils auraient une forme humaine, le corps noir mais le ventre blanc et la peau couverte de chenilles urticantes. Pour compléter le tableau, nos personnages seraient dépourvus d'anus, mais dotés d'un pénis démesurément long et qu'ils sont obligés de porter noué autour du cou (thème classique de la mythologie pano). Les épines (musha) ne pénètrent pas leurs pieds. Pour qui dispose d'un minimum d'imagination structuraliste, ce trait renforce l'idée qu'ils incarnent bien l'antithèse des mariwin, ces derniers étant systématiquement mis en rapport avec les épines, celles des palmiers daratsintuk (Astrocaryum muru muru) utilisées pour les fouets ou celles des palmiers wani (Bactris gasipaes) servant pour les tatouages (également désignés par le terme de musha).

En matière d'originalité, la technologie des maru ne le cède en rien à leur morphologie : en guise d'assiettes (ancha), ils utilisent des raies (ihi), pour les sièges (tsate), des caïmans (kapët) et en guise de bâtons à fouir (mekte), des gymnotes (dendu). Outre ces animaux-accessoires pour le moins insolites, la ménagerie des maru comprend également des ouistiti (sipi) ${ }^{9}$ et des pénélopes ( $k w e ̈ b u)^{10}$. Ils élèvent aussi des jaguars (kamun) qu'une illusion perspectiviste les amène à percevoir comme autant de pécaris à lèvres blanches ${ }^{11}$. Ces félinssuidés se montrent si dociles et si bien familiarisés qu'on peut leur toucher les dents. C'est cependant le pécari à collier (unkin) qui est le principal animal domestique (wiwa) des maru, puisqu'ils le voient comme un chien (wapa). En revanche, il m'a été explicitement précisé que les esprits n'ont pas de "vrais » pécaris à lèvres blanches (chawa), sans doute parce que la «niche » est déjà occupée par les jaguars (kamun). Les kamun étant leurs chawa, quelle place pourraient-ils encore accorder aux vrais chawa?

Le lien privilégié entre les maru et les pécaris à collier, associé au déni explicite de toute relation réelle entre ces êtres et les pécaris à lèvres blanches, permet de supposer qu'il existe une union étroite entre ces derniers et les mariwin. Ce serait, d'une part, conforme au principe de distribution complémentaire qui régit les relations entre les deux entités spirituelles, d'autre part, compatible avec l'image des pécaris à lèvres blanches considérés comme bénéfice annexe (ou produit dérivé) de la mortalité humaine. L'idée que ces animaux sont une sorte de retombée positive des décès survenus dans son propre groupe (ou parfois chez des voisins) semble fort répandue en Amazonie (y compris chez les Kulina dont on reparlera ci-après, voir Pollock 1993).

Dans cette optique, les jaguars - qui tiennent lieu de pécaris à lèvres blanches pour les maru -, renverraient tout au contraire aux thèmes de la malemort et des 
aspects négatifs de la réincarnation ${ }^{12}$. Les Matis établissent une concordance entre leurs morts et les pécaris à lèvres blanches, en affirmant que ceux d'entre eux (supposés être la majorité) qui décèdent lors de la saison des pluies (celle aussi des mariwin) résident dans un inframonde où ces animaux abondent. Relevons enfin que l'image du pécari à collier comme doublet atténué, avatar faible, du pécari à lèvres blanches est un thème très classique de la mythologie amazonienne (Calavia Sáez 2001) ; que ce schéma vienne se plaquer sur l'opposition maru/mariwin n'a donc rien pour nous surprendre.

Une dernière caractéristique des maru mérite d'être signalée : la poudre obtenue à partir de leurs os calcinés est censée conférer des pouvoirs magiques. Elle permet de faire disparaître chez autrui et réapparaître chez soi tout objet convoité. Quiconque a la chance de pouvoir tuer un maru (ou d'en récupérer un cadavre) peut détourner à son profit la faculté de « faire disparaître ». On a jadis signalé d'autres récupérations à des fins sociales (quoique égoïstes) des propriétés normalement néfastes des maru, y compris dans le cadre de l'ornementation corporelle ${ }^{13}$ (Erikson 1996, p. 231). Rien de bien exceptionnel, donc, dans cette poudre d'os de maru, si ce n'est qu'elle rappelle une fois de plus en filigrane leur ressemblance avec les mariwin, dont les masques (autant dire les os) font également partie des choses que l'on retrouve calcinées (en l'occurrence, dans les jardins). Le rapprochement est d'autant plus frappant que les cendres provenant de la crémation d'entités spirituelles apparaissent souvent, dans l'Ouest amazonien, comme l'origine mythique de nombreuses plantes médicinales. Or les entités spirituelles en question - les yoshin shëtaya shipibo, par exemple - sont apparentées aux maru et/ou mariwin. Voilà qui jette sans doute une lumière nouvelle sur certaines pratiques telles le phanéro-cannibalisme shipibo (consistant à brûler les cheveux de ses morts pour en manger les cendres) ou encore le fait que les fougères qui recouvrent le corps du mariwin soient appelées son dawë, son « remède végétal ».

Résumons. Les mariwin s'opposent aux maru, comme la séniorité fertile à l'immaturité stérile ou encore le surhumain à l'infra-humain. Transposées dans un registre ontologique, ces caractéristiques incarnent deux pôles, l'« avant la vie » et l'« après la vie », encadrant symboliquement le déroulement temporel de l'existence humaine. Les ressemblances entre mariwin et maru s'expliqueraient alors par leur appartenance commune à l'univers du " non-vivant », soit par excès, soit par défaut. Laissons les maru, incarnations de destins inaccomplis, à leur triste sort et voyons à présent dans quelle mesure les traits spécifiques des mariwin leur confèrent des propriétés susceptibles de les faire passer pour une métaphore poignante d'un passé hautement idéalisé, autrement dit pour une image de l'ancestralité. 


\section{MARIWIN ET ANCESTRALITÉ}

Les raisons qui permettent de penser que les mariwin sont une représentation de l'ancestralité sont multiples et concordantes. Soulignons tout d'abord qu'il ne fait aucun doute que les mariwin soient des morts. Ils sortent de sous la terre couverts de boue - pareils à des cadavres fraîchement déterrés - et ils ne portent pas de colliers piskare, ornements dont on a montré le lien avec la maturité sexuelle et la communauté des vivants (Erikson 1996). Savoir si les mariwin sont plus spécifiquement des morts ayant anciennement appartenu au groupe est une question plus délicate, sur laquelle nous reviendrons. Relevons cependant d'emblée que les parents défunts s'insèrent dans le même ensemble que les mariwin et les maru. Ces trois types d'êtres sont les principaux destinataires des cris, hautement conventionnels, poussés par les femmes ménopausées chez les Matis (Erikson 2000a). Or ces pratiques laissent transparaître une symétrie dans laquelle le pôle opposé aux maru n'est plus celui des mariwin, mais plutôt celui des défunts. En effet, on fait appel aux maru pour éloigner l'orage et aux morts pour, au contraire, faire pleuvoir ${ }^{14}$. Les défunts et les mariwin semblent donc interchangeables quand il faut agir comme pendant structurel aux maru. Ceux que l'argument ne convainc pas retiendront du moins qu'on s'adresse à ses parents défunts de la même façon qu'aux mariwin (et, accessoirement, aux maru).

Dans une optique comparative, le caractère "ancestral» des mariwin se dégage également de leur similitude avec les esprits que l'on retrouve chez leurs voisins et qui sont explicitement présentés comme des émanations de leurs propres aïeux. Je pense en particulier aux fondateurs mythiques des sections marubo - dont la ressemblance avec les mariwin a été finement analysée par Melatti (1992) - ou aux revenants noshman des Matses ${ }^{15}$. Sans doute faut-il également se référer aux " esprits de l'œil » (bëro yushin, etc.) qui sont connus dans toute l'aire pano, chez les Shipibo, les Cashinahua, les Yaminahua, les Marubo ou les Katukina. Ces êtres sont une des composantes éternelles de la personne qui continue à surveiller ses descendants depuis l'au-delà. Même s'ils n'ont pas vraiment d'équivalents chez les Matis, on est frappé par le fait que ces derniers insistent, d'une part, sur la remarquable brillance de l'œil du masque des mariwin, d'autre part, sur leur regard constamment fixé sur les jeunes. Rappelons que les mariwin font en quelque sorte figure d'" exosquelettes ambulants » (voir supra) ce qui, même en Amazonie, n'est pas sans évoquer les thèmes de la continuité intergénérationnelle, notamment dans un contexte de patrifiliation (Chaumeil 1997). Or les systèmes sociaux des Pano septentrionaux présentent une inflexion patrilinéaire assez marquée (Erikson 1996, chap. 6) ${ }^{16}$.

Pour revenir à l'ethnographie matis, notons que les mariwin entretiennent un lien quasi consubstantiel avec les personnes âgées du groupe, laissant entendre qu'ils figurent l'aboutissement du processus de vieillissement. Les discours matis soulignent le fait qu'il s'agit d'aînés (darasibo) et, même, d'aînés superlatifs 
(darasibo kimo, « aînés paradigmatiques »). Les mariwin sont également qualifiés de tsusi, terme pouvant se traduire par " esprit », mais servant aussi à désigner les ombres et surtout les personnes âgées. Ce caractère d'aînesse est essentiel, dans la mesure où la transmission d'énergie par voie d'injections se réalise toujours d'aîné à cadet. Lorsque, par exemple, les hommes se fouettent entre eux pour améliorer leurs performances cynégétiques, ce sont toujours les deux plus âgés qui manipulent les fouets pour transférer du sho aux plus jeunes avant de se fustiger l'un l'autre. Ils utilisent, pour ce faire, les mêmes badines (kweste) de palmier daratsintuk que les mariwin, se comportant de la sorte comme eux.

Un autre indice de convergence entre les personnes âgées et les mariwin est mis en relief dans la croyance selon laquelle seul l'aîné absolu d'un village (le plus âgé des hommes, darasibo kimo) peut, sans danger et grâce au chant de kanchi buntak, « jeune pousse d'ananas », convier les mariwin à venir chez les humains ${ }^{17}$. Tout homme peut inviter les mariwin avec une trompe masën, mais si un jeune s'avisait de le faire en chantant, il risquerait d'être piqué par un serpent en forêt, risque d'autant plus grand, m'a-t-on dit, que les jeunes vont chasser beaucoup plus souvent que les vieux. Cette interprétation quelque peu utilitariste de la croyance n'empêche pas l'ethnologue de penser que l'aîné des hommes, du simple fait que la proximité de la mort va en augmentant à mesure qu'on avance en âge, se trouve naturellement plus « proche » des mariwin que ses compagnons moins âgés. Les uns sont pour ainsi dire des " vivants déjà morts » (que l'on appelle d'ailleurs tsusi, comme s'ils étaient déjà des fantômes), tandis que les autres seraient plutôt des sortes de « morts encore vivants ». Leurs trajectoires se rejoignent.

La ressemblance entre vieillards et esprits se dégage également de l'observation des prohibitions alimentaires. Une fois les jeunes tatoués, ils doivent réintroduire progressivement les différentes viandes dans leur alimentation, selon un ordre fixe et immuable. Or si la plupart des gibiers redeviennent assez rapidement autorisés, la viande d'ara - nourriture de prédilection des mariwin - n'est en revanche accessible que lorsqu'on atteint le troisième âge : elle ne peut être mangée que par ceux dont les tatouages sont tellement vieux qu'ils ont l'air pratiquement effacés ${ }^{18}$.

Le lien entre les mariwin et les aras s'explique, entre autres, par la longévité de ces derniers, ainsi que par leur fréquentation des mêmes lieux (anciens jardins, falaises). De plus, selon au moins un de mes interlocuteurs (Erikson 2002), les marques faciales de ces oiseaux présentent de fortes similarités avec les tatouages des Matis. Si les mariwin sont toujours aussi nombreux aux cérémonies d'imposition des tatouages, sans doute est-ce donc parce qu'ils sont kwenat kurasek, « jaloux des aras ».

Les Matis répètent à qui veut l'entendre que leur impressionnante ornementation faciale a pour vocation principale d'accroître leur ressemblance avec les mariwin, proclamant tantôt que leurs ornements les rendent pareils à ces esprits, tantôt qu'ils les rapprochent de leurs anciens (darasibo) qui, eux, ressemblaient 
aux mariwin plus encore que les Matis contemporains. Les tatouages sont par ailleurs des occasions au cours desquelles l'accent est mis sur l'imitation des anciens et la succession des générations. Avant de se faire tatouer, les jeunes doivent d'abord demander à l'exécutant, sous le regard attentif des mariwin: "fais-le moi maintenant, comme on te l'a jadis fait à toi » (Erikson 2003). On pourrait donc penser que les Matis se font tatouer pour imiter leurs ancêtres et que cette similitude est médiatisée par les esprits. Reste cependant le paradoxe, déjà évoqué, que les tatouages ne sont guère l'ornement principal des mariwin, puisque leur masque met l'accent sur les innombrables labrets autour de la bouche et les longues rectrices d'aras sur le haut des lèvres. Cela est assez troublant dans la mesure où on a déjà montré que, si le tatouage connote bien l'alliance, les labrets (et, en particulier, ceux du haut du visage) renvoient en revanche à la thématique de la consanguinité, s'opposant de la même façon que ce qui vient de l'oncle maternel et ce qui tient du père (Erikson 1996). On en déduit que, si la célébration de l'alliance (la cérémonie des tatouages dans des jardins neufs) permet de mobiliser les mariwin, les ornements qu'ils exhibent n'en marquent pas moins la consanguinité. Ainsi, le prétexte qui suscite la venue des mariwin est un rituel centré sur l'aftinité, alors que la logique qui l'imprègne repose sur des principes de filiation patrilinéaire.

Relevant des $t$ susi (selon les contextes, " ombre », « esprit » ou « vieux »), les mariwin sont intimement associés à la mort, l'ancien temps et la perpétuation des identités collectives par imitation d'une aînesse hautement respectée. Ils occupent les jardins abandonnés - autrement dit les sites des générations passées - et ils jouent un rôle prééminent dans les rites de passage. La notion de résurrection, liée en particulier aux cycles agricoles, domine dans les discours qui les concernent et qui laissent également une place importante à l'imitation des anciens et à la succession des générations. On le voit, toutes les conditions semblent bel et bien réunies pour qu'on puisse insérer les mariwin dans la catégorie des ancêtres. Cette interprétation rencontre cependant un obstacle majeur : les Matis nient explicitement le fait que lesmariwin soient leurs parents, les présentant plutôt comme leurs tawari.

\section{NoS AMIS LES TAWARI}

Tawari est le terme systématiquement utilisé par les esprits pour s'adresser aux Matis. Lorsque les mariwin rendent visite aux humains, les grognements qu'ils profèrent en arrivant sont censés signifier : "nukun tawari eobi isek », " je viens voir mes tawari », et s'ils ont cessé de venir après les épidémies post-contact, c'est, dit-on, parce qu'ils avaient de la peine pour leurs tawari et leurs tawarin baku (les enfants de leurs tawari). D'après Tumi Preto, il serait plus approprié que les mariwin appellent les Matis nukun igbo (« mes maîtres »), plutôt que nukun tawari (« mes tawari »). Tawari n’en demeure pas moins le mot qu'ils emploient. 
Pour m'expliquer la signification de ce terme, les Matis évoquaient une relation proche, entre personnes qui se ressemblent et s'apprécient, mais sans être apparentées. Ils me donnèrent en exemple les liens qui m'unissaient avec un réalisateur anglais lors du tournage d'un documentaire - ironiquement intitulé « Return of the ancestors ». Ils savaient que nos lieux de résidence et nos langues maternelles respectives étaient différentes, mais voyaient aussi qu'on passait le plus clair de la journée à travailler ensemble. Nous étions donc tawari l'un pour l'autre.

En première approximation, on peut donc traduire tawari par « ami », soit une relation entre personnes qui ne sont pas préalablement apparentées et appartiennent à des groupes différents. Les Matis qui s'entendent particulièrement bien entre eux ne sont pas qualifiés de tawari. En revanche, lors des premiers contacts pacifiques avec la Funai, au milieu des années 1970, la toute première question posée à un fonctionnaire, rencontré alors qu'il revenait de la rivière, a été : " autsi korokanon mibi wakarapa wek, tawari? 》 (" c'est pour cuisiner quoi que tu rapportes de l'eau, tawari? »).

Les mariwin seraient certes des amis, mais aussi des étrangers, et surtout pas des parents. Cela est d'ailleurs parfaitement cohérent avec le fait que les coups de fouet dispensés en vue d'augmenter la fertilité le sont, en règle générale, par des affins (l'oncle maternel fouette par exemple les femmes au moment de leur ménopause). De plus, certains hommes, lorsqu'ils incarnent les mariwin, refusent de fouetter les enfants dont ils sont trop proches, se concentrant plutôt sur leurs parents croisés. Peut-être faudrait-il, pour définir les mariwin matis, utiliser l'oxymore « ancêtres par alliance ». À moins que l'expression paradoxale d' « ancêtres naturalisés » soit préférable pour rendre compte de la nature « étrangère » de ces esprits.

Je n'ai jusqu'ici repéré, dans les autres langues pano, qu'une seule autre occurrence du mot tawari. Dans le récit intitulé «Uma guerra », l'historien brésilien, Capistrano de Abreu (1914, p. 64, énoncé $\left.n^{\circ} 491\right)$, rapporte les paroles d'un Caxinauá qui appelle son adversaire Cuntanauá (Pano), " tawarĩ ». On retrouve là notre " tawari » puisqu'en Caxinauá, la nasalisation finale renvoie simplement au vocatif. L'informateur d'Abreu traduit le terme par « inimigo », mais sans doute le terme détermine-t-il une relation moins franchement marquée par l'hostilité, puisque le Caxinauá qui l'utilise s'efforce d'amadouer et/ou de tromper son adversaire : " inimigo, eu te atirei não, me atira não! » (« ennemi, je ne t'ai pas tiré dessus, ne me tire pas dessus »). Tawari se présente en fait comme une alternative à des termes certainement plus hostiles, tels que " txai » (beaufrère) ou "nawa » (étranger).

S'il est rare dans les langues pano, tawari semble en revanche courant dans celles de leurs voisins de la famille Katukina, où il signifie précisément le contraire d'« ennemi ». Ainsi, en Kanamari (langue de la famille Katukina), tawari veut dire " ami » et peut s'employer dans certains contextes pour se référer à des 

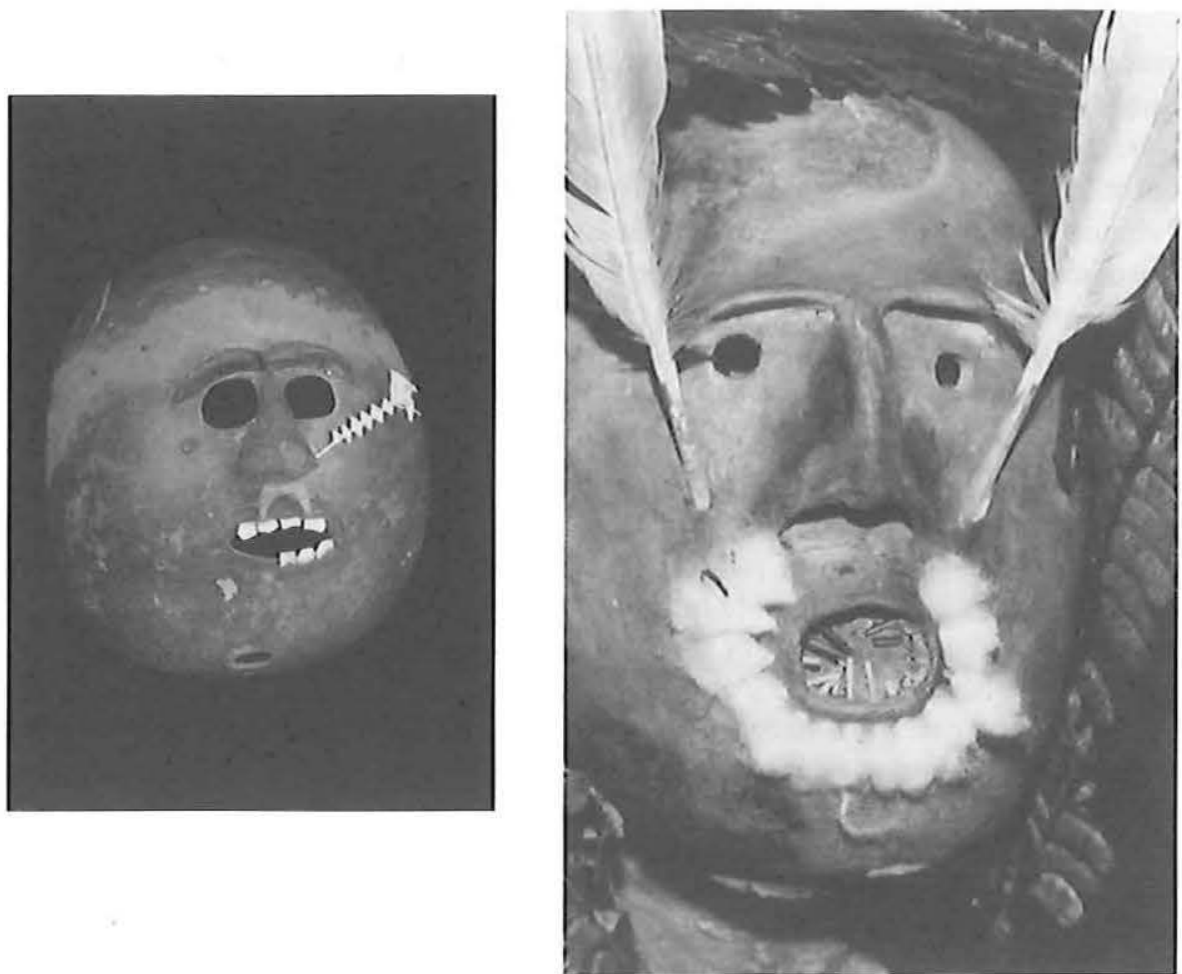

FIG. 3a - Masque Katukina rapporté par Tastevin, Brésil-Amérique $\mathrm{xx}^{\mathrm{e}}$ siècle. Calebasse, résine, plume, nacre, laiton Omusée du quai Branly, Paris - dépôt du Muséum d’hisroire naturelle, musée de l'Homme

FIG. 3b - Masque matis. Céramique, plume, kapok, fougères (cliché Erikson)

groupes locaux (djapa) dont on se sent proche (Reesink 1993, p. 26). Cette homologie est peut-être due au hasard, simple aléa de la combinatoire phonologique. Cependant, l'hypothèse d'un emprunt au Kanamari-Katukina mérite de retenir notre attention parce qu'elle permettrait de comprendre l'énigme suivante : de tous les masques ouest-amazoniens, les seuls qui ressemblent à ceux des Matis sont précisément ceux des Katukina (voir d'Harcourt 1948 ; Labiak 1997). Ces masques offrent en effet des traits harmonieux, ont de belles dents et de jolis ornements ; ils diffèrent en cela de ceux des Pano (Matis exceptés) qui forment des êtres grotesques, hirsutes et à la dentition effarante. Ainsi, les masques matis semblent prendre le contre-pied systématique de ceux des autres Pano (Erikson 2002) et présentent en revanche de troublantes similitudes formelles avec ceux des Katukina (Figure 3). 
Les rares données ethnohistoriques dont nous disposons sont parfaitement compatibles avec cette dernière remarque. Marcoy $(1867$, p. 116) nous apprend que les Mayoruna du Javari (probables ancêtres, entre autres, des Matis contemporains) étaient « liés d'amitié » avec diverses ethnies (non-pano) de la région entre le Jandiatuba et le Jutahy, ce qui leur permettait d'y circuler librement. L'iconographie montre que ces populations soignaient tout particulièrement l'ornementation du pourtour de leur bouche. Bien que Marcoy mentionne uniquement les Culinos, les Marahuas et les Huaraycus, il est possible que cette zone ait également été occupée par des "Catukina » parmi lesquels Métraux (1948, p. 663) compte les Tawari, installés « between the headwaters of the Jutai River and San Felipe on the Juruá River (lat. $6^{\circ} 30^{\prime}$ S., long. $70^{\circ} \mathrm{W}$ ) ", autrement dit immédiatement à l'est du territoire traditionnel des Matis. Tastevin (1924, p. 423) nous informe de l'existence de plusieurs clans de «katukina » dont un s'appelle les Tawari (terme qu'il traduit par " oiseaux caciques, camarades »), tandis qu'un autre, celui des Ben-dyapa, « habitai[t] autrefois la rive droite ou les affluents de l'Itecoahy, tributaire du Javary ». La probabilité pour qu'ils aient rencontré les ancêtres des Matis ou des Korubo est assez forte. Par ailleurs, des quatre mots de « Canawary » que nous livre Chandless (1866, p. 118), pas moins de trois sont de parfaits cognats de leurs équivalents pano: wari pour "sum», chi-i pour " fire » et wáka pour " water ». Etant donné qu'il s'agit de langues appartenant à des familles totalement distinctes, on peut sans doute voir là une preuve supplémentaire de contacts étroits. Tout cela encourage à penser que les ancêtres des Matis ont très bien pu connaître une époque où la visite de leurs " amis tawari » ne prenait pas systématiquement la forme d'une mascarade.

\section{Conclusion}

Les Matis pourraient être surpris, et peut-être même indignés, de m'entendre suggérer que leurs masques reflètent une époque où l'initiation des jeunes faisait appel à des relations d'alliance interethnique plutôt qu'à la logique endotique qui semble dominer aujourd'hui (et sans doute leur stupeur serait-elle partagée par les Kulina qui fouettent leurs consanguins pour les transformer en affins; voir Pollock 1985). Cependant, si l'on prend cette hypothèse au sérieux, les mariwin seraient, du point de vue matis, «les descendants des aïeux des amis formels de nos aïeux », plutôt que directement "nos aïeux ». Voilà peut-être pourquoi les contemporains ne deviennent plus des mariwin après leur mort, alors qu'il m'a été dit que les humains du passé subissaient cette transformation ${ }^{19}$. Quoi qu'il en soit, les mariwin servent, en dernière instance, de prétexte à un discours sur la consanguinité bricolé à partir de relations d'aflīnité, comme pour mieux illustrer le primat de cette dernière catégorie dans les logiques sociales amazoniennes, où l'aftinité semble bien constituer le pôle non marqué à partir duquel la consangui- 
nité serait constamment obligée de se reconstruire (Viveiros de Castro 2000). Les Matis ne sont d'ailleurs pas les seuls à mettre en scène des relations d'affinité pour tenir des propos qui concernent le rapport à leur propre passé. On retrouve ce même jeu entre écarts spatiaux et écarts temporels chez les Waiwai, par exemple (Howard 1993).

Attachement au passé, relation avec les défunts, volonté de ressembler à ses ascendants, insistance sur la continuité entre les générations : toutes les conditions étaient réunies pour assurer l'émergence, chez les Matis, d'« ancêtres » au plein sens anthropologique du terme. Les notions d'altérité constituante et de coupure ontologique entre vivants et morts - notions dont on connait l'importance dans un contexte amazonien - ont cependant teinté leur conceptualisation du passé avec suftisamment de prégnance pour l'entraîner dans une voie différente, où l'ancestralité se voit en quelque sorte subsumée par des reliquats d'alliances. Les mariwin s'assimilent donc moins à des ancêtres stricto sensu, qu'à une sorte d'émanation résiduelle de la sociabilité des générations antérieures. Si leur lien avec les ascendants des Matis contemporains ne fait aucun doute (d'où l'emphase paradoxale sur leurs labrets au détriment de leurs tatouages), ils sont pourtant formellement exclus de la sphère consanguine. En d'autres termes, les mariwin personnifient une forme d'ancestralité médiatisée qui, de manière typiquement amazonienne, se définirait moins sur le mode de la descendance que sur celui de l'amitié cérémonielle et de l'alliance. Si c'était à refaire, sans doute faudrait-il chercher, pour la monographie que j'ai jadis consacrée à mes tawari matis, un titre plus franchement amazoniste que "La Griffe des aïeux ». *

* Manuscrit reçu en janvier 2004, accepté pour publication en mars 2004.

\section{NOTES}

Cet article a été écrit à l'occasion du symposium : « History and Historicity in Amazonia. Time experienced, conceptualized and enacted ", organisé par Carlos Fausto et Michael Heckenberger pour le 98th annual meeting of the Amerian Anthropological Association, Chicago, 1999.

1. Toutes mes données ethnographiques ont été recueillies entre 1985 et 1988, au cours d'une mission de douze mois financée par une allocation du ministère de la Recherche, une bourse de la fondation Fyssen et des crédits du Centre national de la recherche scientifique. Trois séjours plus brefs (un peu plus de quatre mois en tout) ont été réalisés en 1996, 1998 et 2000, pour le tournage de documentaires. Ces séjours m'ont permis d'obtenir les informations présentées ici. Elles viennent compléter les publications antérieures consacrées aux mariwin (Erikson 1990a, 1990b, 1992, 1996, 2000a, 2001).

2. Selon Romanoff $(1984$, p. 237 sq.), la « sueur » et la «chaleur» seraient, chez les Matses, synonymes de cette forme « d'énergie » que les humains peuvent se transmettre au moyen de substances, telles que le frog skin emetic ou le tobacco powder.

3. Pour une filmographie relativement exhaustive, voir Erikson (2000b, pp. 285-287). Les représentations destinées à la caméra font généralement intervenir plusieurs types de mariwin à la fois (un ocre et deux noirs, par exemple), comme pour fournir un échantillon plus représentatif, à défaut d'être 
conforme aux pratiques anciennes. On est loin de l'époque où les Matis pouvaient dire que les mariwin ne portaient pas de culottes parce qu'ils ignoraient tout des Blancs !

4. Contrairement à ce que j'ai publié dans le passé (Erikson 1996, p. 224), la résine en question est celle qui sert à l'éclairage et non celle qui s'utilise pour enduire les sarbacanes, la confusion provenant de ce que toutes deux sont appelées du terme générique de mamõ.

5. Cette inversion des perspectives évoque le domaine des morts : " there are many reports [from other societies] of the belief that the state of death is in various particulars a continuation of life under an opposite sign, death being conceived of among mumerous African and Indonesian societies as being a state of reversal (up becomes down, front becomes back, etc.) " (Needham 1967, pp. 430-431).

6. Tumi Preto, qui me racontait cela, me fit remarquer que les pécaris à lèvres blanches - qui, selon lui, étaient autrefois des humains - sont naturellement pourvus de deux petits trous de part et d'autre du haut du crâne, très comparables aux orifices des masques de mariwin, ainsi qu'aux foramen de l'os maxillaire des humains, qui se trouvent somme toute juste en-dessous des labrets de leur lèvre supérieure.

7. Ces dents intériorisées contribuent indéniablement à réinsérer les mariwin dans le cadre plus général des mascarades ouest-amazoniennes, puisqu'une dentition impressionnante semble constituer une des caractéristiques principales des masques d'autres groupes arawak et pano de la région, au point de transparaître dans la désignation même du masque munti xëtaya (" calebasse dentée ») des Cashinahua (Kensinger 1975) ou encore du masque yoshin shëtaya (" esprit denté ») des Shipibo, ce dernier ayant en outre la particularité d'être chauve, comme les maru (Loriot et al. 1993 ; Baer 1993).

8. Les pendants d'oreille matis sont blancs et ronds, ce qui les rend semblables à certains champignons. Sur la propension à rapprocher les champignons sauvages et les esprits asociaux, consulter Blust (2000).

9. La description qui nous a été faite du maru comme ayant un corps noir mais un ventre blanc fait penser à certains singes sipi. David Fleck (com. pers.) m'a indiqué que les Matses distinguent quatre espèces de " sipi » dont une s'appelle précisément madum sipi (" demon's tamarin », Cebuella pygmaea), le singe du « maru ». Les Matis évoquent également l'existence d'un animal qu'ils appellent marum sipi, mais il semblerait qu'il s'agisse d'une " espèce » caractérisée par son extrême docilité (ils ne fuient pas) plutôt que par sa morphologie. Par ailleurs, personne ne les aurait jamais vus de près. Seuls les anciens ont eu l'occasion d'observer des marun sipi.

10. Cette insistance sur l'apprivoisement par les maru de vrais $k w e ̈ b u$ redouble d'intérêt car cet oiseau est précisément celui que les mariwin voient dans leurs doublets aviens, les aras. Si on ne le savait pas déjà, on pourrait deviner que le $k w e ̈ b u$ est le premier oiseau introduit dans l'alimentation tandis que l'ara est, au contraire, le dernier.

11. Certaines illusions auxquelles succombent les maru relèvent clairement de ce qu'il est désormais convenu d'appeler le perspectivisme (Viveiros de Castro 1996). On constate cependant qu'une partie des pratiques imputées aux maru défie non seulement l'entendement ordinaire des humains, mais également les lois de la physique. Car ce n'est pas uniquement le regard, mais aussi le postérieur du maru qui est censé se poser sur un caïman-siège. Ce ne serait donc pas seulement la manière de le percevoir, mais le fonctionnement même du monde qui serait ici remis en question (hypothèse multi-naturaliste poussée à l'extrême). À moins qu'il ne s'agisse simplement de souligner, non sans humour, l'impuissance fondamentale propre aux maru (voir infra), dont on imagine bien les piètres résultats qu'ils obtiennent avec leur " bâton à fouir » tout mou.

12. Chez les Capanahua (Loos 1960), ce sont les âmes des mauvais chamanes qui se transforment en jaguars. Bien que les Matis, qui n'ont plus de chamanes attitrés, ne partagent pas cette croyance, on peut néanmoins remarquer que, au cours des semaines qui ont suivi la mort accidentelle (par morsure de serpent) d'un de leurs leaders les plus influents, on s'est mis à déceler régulièrement de nombreuses traces de jaguar aux alentours du village.

13. On se grime en maru, en enlevant ses ornements et en se peignant en noir, notamment à l'occasion d'expéditions guerrières. La pratique rappelle celle des Desana qui, lors d'un rituel destiné à empêcher leurs adversaires de réagir, se peignaient autrefois des croix sur le visage avec la « poudre de 
violence ", cendres supposées provenir de la crémation des os du héros mythique Yurupari et souflées par les chamanes (Dominique Buchillet com. pers.).

14. Les Matis disent que la pluie leur est envoyée par les morts pour boire et se laver. Cela va de pair avec l'idée que la pluie est bénéfique (elle procure de l'endurance). Les maru, pour leur part, sont appelés par les femmes âgées lorsque l'orage menace. Il leur est demandé d'égarer la pluie (de lui faire perdre son chemin) comme ils le font avec les gens en forêt.

15. Les noshman, que Fields (1973) définit comme des revenants incarnant des morts du groupe et qui viennent initier les jeunes, présentent d'assez troublantes ressemblances avec les mariwin matis. Il serait toutefois hasardeux de les confondre totalement, dans la mesure où les Matis reconnaissent également une catégorie d'esprits, distincte des mariwin, qui s'auto-désignent par noshoman, alors que les humains les appellent kushana.

16. Relevons à ce propos que l'étymologie la plus probable du terme darasibo, qui désigne les aînés, pourrait bien se résumer à " purement masculin " (dara : « corps, homme »; -sibo: " uniquement »). Le nom du palmier dont on tire les fouets et les colliers piskaré et dans lequel on dépose le cordon ombilical des nouveau-nés est daratsintuk, terme qui pourrait se traduire par " homme dénudé » (dara : " corps, homme » ; tsintuk : «nu ») et s'interpréter comme "support neutre " à partir duquel on construit de la sociabilité.

17. Sur ce chant, voir Erikson (1996, p. 264).

18. Voilà sans doute une piste pour répondre à la question : pourquoi les tatouages sont-ils si rarement représentés sur les masques de mariwin? Cela s'explique parfaitement si l'on tient compte du fait que les tatouages s'effacent avec l'âge. Sans doute faut-il aussi considérer que la peau du mariwin étant enfouie dans son tréfonds, par contraste avec ses os apparents, les tatouages ne sont visibles que lorsqu'on les représente sous forme « écorchée ». Le seul masque sur lequel j’ai pu voir des tatouages nettement dessinés (incisés dans l'argile) était de ce type, pourvu de dents de pécari grossièrement enfoncées dans la résine.

19. Retenons toutefois qu'une seule et même expression, " tsusin impak- ", " devenir/incarner un esprit ", s'utilise tant pour se référer au port d'un masque que pour décrire ce qui advient après la mort.

\section{BIBLIOGRAPHIE}

\section{Abreu João Capistrano de}

1914 Rã-txa hu-ni ku-î... A lingua dos Caxinauás do rio Ibuaçu, afluente do Muru, Prefeitura de Tarauacá, Typographia Leuzinger, Rio de Janeiro.

\section{BAER Gerhard}

1993 "Para o melhor entendimento das mascaras Sul-Américanas », in Vera Penteado Coelho, ed., Um século de antropologia no Xingu, Editora da Universidade de São Paulo, São Paulo, pp. 289-309.

\section{BiDou Patrice}

1999 «Des fantômes et des hommes. Une topologie amazonienne de l'inconscient ", L'Homme, 149, pp. 73-82.

\section{BLust Robert}

2000 " Rat ears, tree ears, ghost ears and thunder ears in Austronesian languages ", Bijdragen tot de Taal-, Land-en Volkenkunde, 1156 (4), pp. 687-706.

\section{Calavia Sáez Oscar}

2001 «El rastro de los pecaríes. Variaciones míticas, variaciones cosmológicas e identidades étnicas en la etnologia pano ", Journal de la Société des Américanistes, 87, pp. 161-176. 
Chandless William

1866 "Ascent of the river Purûs ", Journal of the Royal Geographical Society, XXXV, pp. 86-118.

\section{Chaumeil Jean-Pierre}

1997 «Les os, les flûtes, les morts. Mémoire et traitement funéraire en Amazonie ", Journal de la Société des Américanistes, 83, pp. 83-110.

\section{ERIKson Philippe}

1989 "Les Matis de la tête aux pieds et du nez aux fesses », in M.-L. Beffa et R. Hamayon, éds, Les Figures du corps, Société d'ethnologie, Nanterre, pp. 287295.

1990a "How crude is Mayoruna pottery? ", Journal of Latin American Lore, XVI (1), pp. 47-68.

1990b "Near beer of the Amazon », Natural History, 90 (8), pp. 52-61.

1992 "Poils et barbes en Amazonie indigène : légendes et réalités », Annales de la Fondation Fyssen, 7, pp. 83-91.

1993 "A onomástica matis é amazônica ? ", in E. Viveiros de Castro e M. Carneiro da Cunha, eds, Amazônia : etnologia e historia indigena, Núcleo de História Indígena e do Indigenismo, USP/FAPESP, São Paulo, pp. 323-338.

1996 La Griffe des aïeux. Marquage du corps et démarquages ethmiques chez les Matis d'Amazonie, Peeters/SELAF, Louvain/Paris. [traduit en espagnol en 1999, El Sello de los antepasados. Marcado del cuerpo y demarcación étnica entre los matis de la Amazonía, Abya Yala, Institut français d'études andines, Quito/Lima.]

2000a " "i", "uuu", "shhh"...»: gritos, sexos e metamorfoses entre os Matis (Amazônia Brasileira) », Mana, 6 (2), pp. 37-64. [version française in Alain Babadzan, éd., Insularités. Hommage à Henri Lavondès, Société d'ethnologie, Nanterre, 2003, pp. 197-228.]

2000b «Bibliografia anotada de fuentes con interés para la etnología y ethnohistoria de los pano setentrionales (matses, matis, korubo...) ", Amazonía Peruana, 27, pp. 231-287.

2001 «Myth and material culture: Matis blowguns, palm trees, and ancestor spirits ", in Laura Rival and Neil Whitehead, eds, Beyond the visible and the material: the amerindianization of society in the work of Peter Rivière, Oxford University Press, Oxford, pp. 101-121.

2002 " Le masque matis. Matière à réflexion, réflexion sur la matière ", L'Homme, 161, pp. 149-164.

2003 " "Comme à toi jadis on l'a fait, fais-le moi à présent...". Cycle de vie et ornementation corporelle chez les Matis (Amazonas, Brésil) », L'Homme, $167-168$, pp. $129-152$.

FIELDS Harriet

1973 "Notes on the "Singing People" ceremony », Información de Campo, 131-d, ms.

Harcourt Raoul d'

1948 Arts primitifs d'Amérique, Éditions du Chêne, Paris. 


\section{Howard Catherine}

1993 «Parawa : a farsa dos visitantes entre os Waiwai da Amazônia setentrional », in Eduardo Viveiros de Castro et Manuela Carneiro da Cunha, eds, Amazônia : etnologia e historia indigena, Núcleo de História Indígena e do Indigenismo, USP/FAPESP, São Paulo, pp. 229-264.

\section{KENSINGER Kenneth}

1975 "Studying the Cashinahua », in Jane Powell Dwyer, ed., The Cashinahua of Eastern Peru, The Haffenreffer Museum of Anthropology, Brown University, pp. 9-85.

LABIAK Araci Maria

1997 "Frutos do Céu » e "Frutos da Terra »: Aspectos da cosmologia Kanamari no warapekom, thèse de mestrado, Universidade Federal de Santa Catarina, Florianópolis.

Loos Eugene

1960 "Capanahua patrilineality and matrilocality », Información de Campo, 67, ms..

Loriot James, E. Lauriault y D. Day

1993 Diccionario shipibo-castellano, ILV, Pucallpa.

Marcoy Paul

1867 "Voyage de l'océan Pacifique à l'océan Atlantique à travers l'Amérique du Sud. Douzième étape. De Tabatinga à Santa Maria de Belen do Para », in Edouard Charton, éd., Le Tour du Monde. Nouveau journal de voyages, Librairie Hachette et Cie, Paris, pp. 97-160.

Melatti Julio Cezar

1992 "Enigmas do corpo et soluções dos Panos », in Mariza Corrêa y Roque Laraia, eds, Roberto Cardoso de Oliveira, Homenagem, UNICAMP, Campinas, pp. 143-166.

MÉTraux Alfred

1948 "Tribes of the Jurua-Purus Basins », in Julian Steward, ed., Handbook of South American Indians. III. The Tropical Forest Tribes, Cooper Square Publishers, New York, pp. 657-686.

MoLINIÉ Antoinette

1988 "Sanglantes et fertiles frontières. À propos des batailles rituelles andines », Journal de la Société des Américanistes, 74, pp. 49-70.

NeEDHam Rodney

1967 "Right and left in Nyoro symbolic classification ", Africa, XXXVII, pp. 425-452.

\section{POLLOCK Donald}

1985 "Looking for a sister : Culina siblingship and affinity », in K. Kensinger, ed., The sibling relationship in Lowland South America, Working Papers on South American Indians, Bennington College, Bennington, pp. 8-15. "Death and the afterdeath among the Kulina », The Latin American Anthropology Review, 5 (2), pp. 61-65. 
REESINK Edwin Boudewijn

1993 Imago mundi Kanamari, tese de doutorado, Universidade Federal de Rio de Janeiro, Rio de Janeiro.

RoMANoFf Steven

1984 Matses adaptations in the Peruvian Amazon, Ph. D. dissertation, Columbia University, New York.

TASTEVIN R.P. Constant

1924 "Les études ethnographiques et linguistiques du P. Tastevin en Amazonie », Journal de la Société des Américanistes, 16, pp. 421-425.

TowNSLEY Graham

1987 "The outside overwhelms : Yaminahua dual organization and its decline », in H. Skar and F. Salomon, eds, Natives and neighbors in South America, Göteborg's Etnografiska Museum Göteborg, pp. 355-376, coll. « Etnologiska Studier » 38.

Viveiros de CAstro Eduardo

1996 "Os pronomes cosmológicos e o perspectivismo ameríndio », Mana, 2, pp. 115-144. [traduit en anglais en 1997 : « Cosmological Deixis and Amerindian Perspectivism ", Journal of the Royal Anthropological Institute, 4, pp. 469-488.]

2000 «Atualização e contra-efetuação do virtual na socialidade amazônica : o processo de parentesco ", ILHA, 2 (1), pp. 5-46. 